\title{
The Spine Crasher: A Challenging Diagnosis
}

Muhamad Azamin Anuar, Mossad Abdelhak Shaban Mohamed, Nur Syuhada Mohd Azhar, Asrar Abu Bakar, Taufiq Hidayat Hasan

Kulliyyah of Medicine, International Islamic University Malaysia, Kuantan, Pahang.

Introduction: Spinal tuberculosis in children is an established preventable disease in developing countries. Complications are devastating due to its aptitude to cause bone destruction, spinal deformity and paraplegia. Case report: We present an eight-month old girl with isolated gross motor regression and evolving spastic paraplegia. It highlights the challenge we encountered due to delay in garnering the pertinent investigation. She presented to clinic with history of legs weakness and loss of rolling after a period of prolonged febrile illness. Both parents are medical practitioners. Mother had history of SVT during pregnancy in spite on anti-arrhythmic treatments. Her father is a thalassaemia carrier. A thorough examination revealed gross motor delay and upper motor neuron signs. She had raised inflammatory markers, anaemia and thrombocytosis with persistent low-grade temperature. CT brain with contrast showed meningeal enhancement. Full septic work up revealed the CSF result reflecting partially treated meningitis. She was treated with third generation cephalosporin and acyclovir. Mother claimed exposure to TB patients hence Mantoux test was recommended which came positive. Her chest x-ray, sputum culture, CSF culture and NAA studies came non-conclusive for pulmonary tuberculosis. MRI for brain and spine showed features of tuberculous spondylodiscitis of T4-T5 vertebrae with associated subligamentous paravertebral spread and epidural extension causing spinal cord compression and T3-T6 hydro-syringomyelia. After multidisciplinary team discussion, patient started on intensive antituberculosis regimen with good initial response. Discussion: Clinically lower limbs power improved with good antigravity movement. Laboratory and radiological investigations have improved inflammatory markers and dropping trend thrombocytosis, and spinal gibbous stay stationary with improvement in plain radiology. She is under regular follow up awaiting serial MRI. Conclusion: The challenge in diagnosis of extra pulmonary tuberculosis in infants is getting them excluded early. High index of suspicion along with radiological investigation is vital to aid the diagnosis and establishment of treatment to expect a good outcome. 